\title{
Analysis on the Influencing Factors and Training Methods of Art Examinees' Artistic Expression of Sports Dance
}

\author{
Qin Fei \\ Zaozhuang University \\ No. 1 Bei'an Road, Shizhong District, Zaozhuang, Shandong
}

\begin{abstract}
The artistic expression of sports dance plays an important role in the examination of dance art examinees and is one of the vital factors affecting art examinees' performance. By means of literature method and logic analysis, this paper probes into the factors influencing art examinees' artistic expression of sports dance, and then discusses the training methods to improve the artistic expression. The study finds that the factors affecting the artistic expression of art examinees are as follows: special basic knowledge, special physical quality, special psychological quality and special music knowledge.
\end{abstract}

Keywords-Sports dance; Art; Expression; Art examinees

\section{INTRODUCTION}

In the 1930s, foreign ballroom dance was introduced to China. In the 1980s, the competitive sports dance was launched in China. In October 1986, China officially introduced sports dance. In the 1980s, sports dance gradually entered the university curriculum project. In 1987, the State Education Commission added sports dance in college physical education major. Since then, it has offered sports dance as an elective course in the university public physical education classes. In 1991, the State Education Commission issued the "Undergraduate Physical Education Major Teaching Plan for General Colleges and Universities", which listed sports dance as a compulsory course for the physical education department of higher normal colleges. The compulsory course of sports dance was deeply loved and favored by the majority of examinees. Beijing Dance Academy opened the discipline of national standard dance in 1992; from 1994 to 1995, two-year college graduates joined the cause of China's national standard dance. The national standard dance discipline opened a fouryear undergraduate class education in 1997. With the continuous deepening of sports dance in China, Beijing Sports University took the lead in setting up sports dance specials in 1998; and then the physical education departments of normal universities and some sports colleges gradually added sports dance specialties. Tianjin, Guangzhou, Chengdu Sports Colleges established dance majors in 2000-2004. Chengdu Sports Institute, Xi'an Physical Education Institute, Shenyang Physical Education Institute, Wuhan Sports College and Beijing Sport University have successively added performance majors. Shanghai Sports Institute has set up a dance choreographer, beginning a separate enrollment of sports dance
[1]. As a special elective course, sports dance has entered a new development period in China's colleges and universities. Up to now, there have been more than 100 schools nationwide that recruit sports dance special projects.

In recent years, there are more and more art examinees for sports dance. There are three reasons: first, sports dance is easier for them to enter the colleges and universities; second, the scores of cultural classes are low; third, the market demand is large. Recent years have witnessed that the regulations of the art test are constantly changing. Previously, attention was paid to professional and technical achievements. Nowadays, the emphasis on cultural courses is more important, and the pressure on sports dance examinees is also growing.

The artistic expression of the sports dance examinees is the main reference for the examiners' evaluation, but most of the sports dance examinees have relatively weak artistic expression, which weakens their performance. Since improving the artistic expression of sports dance examinees requires a long-term process, how to carry out systematic artistic expression training for sports dance examinees has become an urgent problem to the training teachers of various sports dance examinations. Therefore, this research focuses on how to improve the artistic expression of sports dance examinees, and provides a reference for sports dance training teachers.

\section{RESEARCH OBJECT AND METHODS}

\section{A. Research Object}

This study mainly takes the sports dance examinees in the past three years as the research object.

\section{B. Research Methods}

\section{1) Literature Method}

Access and analyze literature and writing related to artistic expression, sports dance, sports training, performance theory, music, etc. Log into relevant project websites and collect relevant information about the 2000-2018 Sports Dance Art Examination to provide a basis for this research.

\section{2) Video Analysis}

The video of the youth group of international and domestic sports dance competitions was observed and analyzed. 


\section{3) On-site Observation}

Carry out on-the-spot observations of the training and examinations of sports dance examinees, and get the first-hand information through field observation.

\section{4) Interview Method}

Consult with instructors who participate in the sports dance admission examinations and coaches of sports dance examinees on the artistic expression of sports dance examinees.

\section{RESULTS AND ANALYSIS}

Through the analysis of expert interviews, combined with the author's teaching experience, and drawing on relevant theories of sports dance by scholars at home and abroad, it is concluded that the sports dance examination pays great attention to the artistic expression of examinees. In teaching and training, the author found that most sports dance examinees have good special skills, but the final test results are not ideal. The reason is that examinees lack the artistic expression of sports dance. Therefore, improving sports dance examinees' artistic expression requires training from four aspects: professional basic knowledge, physical quality, music theory and psychological quality. Based on these four aspects, this paper explores ways to improve the artistic expression of sports dance examinees.

\section{A. Enhance the artistic expression of sports dance examinees by improving professional basic knowledge}

Improve the theoretical knowledge of sports dance examinees from the cultural background: sports dance is divided into two major dance systems: Latin dance and modern dance. Latin dances include Lombard, Cha Cha, Cowboy, Samba and Bullfighting; modern dances include waltz, tango, brisk, foxwalk and viennese waltz. These 10 dances are all standardized by Western folk dances and each dance has an original culture and a distinctive western color. Sports dance is the perfect combination of sports and art, so art examinees need strong expressiveness when displaying. However, the different cultural backgrounds between China and the West have a certain influence on the expressive power of sports dance examinees. In the game, we can feel that the players in the Western countries can more naturally radiate their enthusiasm and their emotions are more casual and free, while the players in our country are more restrained and implicit.

Excellent sports dance examinees, in order to better interpret the connotation of sports dance, need to master the cultural implication of each sports dance and clearly understand its background and style, thus improve the artistic expression of sports dance. .

\section{B. Enhance the artistic expression of sports dance examinees by improving physical fitness}

Sports dance is a high-intensity exercise. The requirements of posture in the art test are rigorous. In the stressful and stimulating test, examinees need to complete complex sets of movements as required, and must have a certain artistic expression of sports dance. Therefore, the requirements for physical fitness are high. To improve artistic expression, examinees need to train their special strength, speed, coordination, endurance, flexibility and control.

\section{1) Special Strength Training}

Sports dance is flexible and complicated. Many movements and stylings require special strength. Without a good special strength foundation, they cannot show the explosiveness and passion of sports dance. Sports dance requires not only the training of leg strength, but also the training of the waist and upper limbs. For the legs, you can bounce and run, so as to improve the muscle strength of the legs. For the waist and abdomen, you can use v-up, the flat support, the sit-ups and enhance the back muscles. For the upper limbs, you can perform push-ups, dumbbells, etc. [2].

\section{2) Special Speed and Coordination Training}

The coordination of examinees is one of the important factors affecting the performance of sports dance. Sports dance is a set of combinations that require examinees to complete a specified number of movements within a period of time, and also to reasonably cut the music rhythm or "fast" and "slow" to achieve perfect coordination with music. Speed and coordination training is required to complete the above requirements. For the speed quality, they can use the elastic band for auxiliary training in basic training or a complete set of exercise; for the coordination quality, they can carry out training such as steering jump, cross jump and single leg jump, and combine the speed and coordination training. In this way can the quality of speed and coordination be better improved.

\section{3) Special Endurance Training}

Examinees' specific endurance determines the quality and skill of the action. Sports dance itself is a long-lasting exercise of the body. The energy consumption of the body is huge. Without good special endurance, in completing a series of high-intensity exercises in the exam, the examinees' artistic expression of sports dance will have the decline. Good special endurance is the basic special quality of sports dance examinees.

In the special endurance training, the continuous practice method can be used first. The coach organizes the examinees to perform the combined exercises without interruption. This practice method can improve the endurance of the examinees; then use the intermittent practice method so that the body can be recovered between the groups. This training effect is slightly better than the continuous training method.

\section{4) Special Flexibility Training}

In the colleges that recruit sports dance specialties, the examination covers flexibility, but there are very few examinees who can complete this standard. Not only is there flexibility in the exam, but also special flexibility is an important part of physical fitness. The special flexibility directly affects the artistic expression of sports dance. If the examinee does not have good flexibility, then the beauty of sports dance cannot be demonstrated. In completing the movement, there is not enough stretch or insufficient amplitude, which affects its expressiveness. The special flexibility can be divided into three parts: the upper limb, the trunk and the lower limb. The upper limb can be shoulder-pressed or shoulderworked with the props; the trunk can be practiced in the lower 
back, flexion and sideways; the lower limb can be pressed, kicked, and moved. Strengthening the special flexible training can make the examinees' movements more beautiful and extended, and finally improve the artistic expression of the sports dance examinees.

\section{5) Special Control Training}

In the examination, examinees must enjoy strong special control ability in order to display wonderful artistic expression in sports dance. The special control of sports dance is mainly controlling dancing postures, including external manifestations through limbs, body torso and head, as well as changes in rhythm, body center of gravity, and range of motion. The action postures of sports dance are divided into static and dynamic, which bring strong visual impact and emotional appeal. Special control training can use the elastic band to train through repeated exercises of a single action, so that the muscles achieve good control [3].

\section{Enhance the artistic expression of sports dance examinees through music theory.}

Music plays an important role in promoting the artistic expression of sports dance examinees. A sports dance student who does not understand music lacks spirituality and essence in his/her sports dance, thus impairing the effect of the whole paragraph. Only when music and dance are combined can a more graceful dance be demonstrated. Sports dance and music are related from outside to inside. Sports dance must have musical accompaniment to better reflect its expressiveness. The strength, speed, flexibility and other aspects during the examinees' examinations are directly influenced by music. The movements of sports dance change along with the changes of music. Music can make examinees more absorbed and devoted, which will boost the artistic expression of sports dances [4].

\section{Enhance the artistic expression of sports dance examinees through psychological quality training.}

1) The psychological factors affecting the artistic expression of sports dance examinees.

Under excessive stress before the test, the examinees will have shortness of breath, restlessness and rapid heartbeat, which will make their technical level decline---they will lose control, the action will become slow, the music and action do not match or they even forget the action.

Examinees' pre-examination psychological training can be arranged according to the different conditions of the examinees. Through the self-examination test, relaxation training method and self-suggestion method, examinees can eliminate the undesirable psychological changes before the test, then form the best test state, and usher in their normal and extraordinary performance.

Psychological training can adopt self-suggestion and relaxation training. Self-suggestion is that the sports dance examinees use their own language and thoughts to make psychological hints to themselves, adjust their negative emotions, and strengthen their emotions as they need. Meditation is a very effective self-psychological suggestion. When examinees are training artistic expression of sports dance, meditation can control their psychological activities very well.
Relaxation training method can make sports dance examinees relieve mental fatigue, and it has a great influence on improving their artistic expression and test scores. Relaxation training can ease tension by listening to music or breathing deeply.

\section{2) Pre-test simulation test training}

The mock exams are arranged according to the form of the sports dance exam and any unexpected situations that may occur in the exam. The simulated exams can bring closer the training and examination of the sports dance artistic expression.

The tactical psychological preparation of the examinees for sports dance has a significant role in the artistic expression of sports dance.

\section{3) Form the best emotional state}

Before the sports dance test, examinees may be affected by various environmental conditions such as the performance of the opponent and the score of the examiner, resulting in abnormal psychological activities and the psychological fluctuations, thus affecting the artistic expression of the examinees. Therefore, the emotional changes of the examinees before the sports dance examination are important psychological factors that directly affect their artistic expression.

\section{4) Establish the confidence}

Confidence is mainly based on the conscious building in the training of the artistic expression of sports dance and on the scientific arrangement before the test. Confidence is an important factor in whether the sports dance examinees can fully exert their artistic expression, for confidence is the belief, the desire or the expectation of a mental state that can be achieved. In addition to constantly strengthening ideological and political education and correcting the motivation of the exam, we should also master the different personality characteristics and will qualities of the examinees, and educate them differently.

The training of psychological quality plays a vital role in improving the artistic expression of sports dance examinees. Even if they have superb skills and physical qualities, without good psychological qualities, they will not be able to display their exquisite skills in the examination.

\section{CONCLUSIONS}

(1) Physical constitution affects the artistic expression of sports dance examinees. The quality of physical fitness directly affects the quality of complete sets of movements.

(2) Psychological quality is one of the important factors affecting the artistic expression of sports dance examinees. It is advisable for examinees to participate in sports dance competitions to train and improve their psychological quality. This is an effective means to exercise the artistic expression of examinees.

(3) Examinees should understand the background of sports dance culture, and at the same time cultivate the musical sense of sports dance examinees, let the examinees understand the music, integrate the music, master the style, characteristics and 
rhythm of the dance and music, so that the artistic connotation of sports dance can be fully displayed.

\section{ACKNOWLEDGMENT}

Address: No. 1 Bei'an Road, Shizhong District, Zaozhuang, Shandong, zip code 271600

Mailbox: 304042923@qq.com mobile phone: 17863261119 QQ: 304042923.

\section{REFERENCES}

[1] Jiang Bingling. Research on the Factors Affecting the Professional Performance of Sports Dance Students [J]. Science Guide, 2015.

[2] Tian Maijiu. Sports Training[M].Beijing: People's Sports Publishing House, 2004.

[3] Tian Bin. Aesthetic Features of Sports Dance [J].Big Stage, 2013

[4] Sun Zhongying, Research on the Method of Training the Performance of Sports Dance Athletes [J]. Journal of Harbin Institute of Physical Education, 2008. 